\title{
Closed-loop power and focus control of laser welding for full-penetration monitoring
}

\author{
Fabrice Bardin, Adolfo Cobo, Jose M. Lopez-Higuera, Olivier Collin, Pascal Aubry, \\ Thierry Dubois, Mats Högström, Per Nylen, Peter Jonsson, Julian D. C. Jones, and \\ Duncan P. Hand
}

\begin{abstract}
We describe a closed-loop control system ensuring full penetration in welding by controlling the focus position and power of a 4-kW Nd:YAG laser. A focus position monitoring system was developed based on the chromatic aberration of the focusing optics. With the laser power control system we can determine the degree of penetration by analyzing the keyhole image intensity profile. We demonstrate performance in bead-on-plate welding of Inconel 718 and titanium. The focus control system maintained a focal position on tilted and nonflat workpieces, and the penetration monitoring technique successfully controlled the laser power to maintain the full-penetration regime in the presence of linear and step changes of thickness. Finally we discuss the performances and the limits of the systems when applied to a realistic complex aerospace component. (C) 2005 Optical Society of America
\end{abstract}

OCIS codes: $140.3390,040.0040,060.2370,100.2960$.

\section{Introduction}

Laser welding offers significant advantages in aerospace manufacture, for both engines and airframes. But in safety-critical applications such as aerospace manufacture it is vital that every weld can be assured to be free of defects, and an on-line process monitoring system is part of the necessary quality assurance process. On-line sensing also offers the possibility of process control, thus reducing the requirement for rework.

Several techniques have been developed for process monitoring and control of laser welding. A small number of optical-based process monitoring

F. Bardin (f.bardin@hw.ac.uk), J. D. C. Jones, and D. P. Hand are with the School of Engineering and Physical Sciences, HeriotWatt University, Edinburgh EH14 4AS, United Kingdom. A. Cobo and J. M. Lopez-Higuera are with the Universidad Cantabria, Avenida de los Castros, s/n, Santander E-39005, Spain. O. Collin is with Snecma Moteurs, RN7, BP 81, Evry 91003 Cedex, France. P. Aubry and T. Dubois are with Cooperation Laser FrancoAllemande, 16 bis avenue Prieur de la Côte d'Or, Arcueil 94114, France. M. Högström and P. Nylen are with the University of Trollhättan/Uddevalla, Gustavamelinsgata 6, Trollhättan 461 29, Sweden. P. Jonsson is with the Volvo Aero Corporation, Trollhättan SE-461 81, Sweden.

Received 12 May 2004; revised manuscript received 24 September 2004; accepted 28 September 2004.

0003-6935/05/010013-09\$15.00/0

(C) 2005 Optical Society of America systems, based on cameras or photodiodes, have been commercialized for several years (for example, Laser Welding Monitor from Precitec, welding monitor PD 2000 from Prometec, ${ }^{1}$ WeldWatcher from $4 \mathrm{D}$, Porosearch/LW, and tracking systems from ServoRobot). In each case it is normal to image the light emitted from the welding interaction region onto the detector. The most elegant solution therefore is to use a suitable beam splitter so that the detector can view the welding region coaxially with the laser light; this approach has been followed by many researchers and companies. ${ }^{1-10}$ On the basis of these techniques, a number of closed-loop process control systems have been realized. Several authors have demonstrated the possibilities of feedback control strategies by using, e.g., the laser power, ${ }^{8-12}$ the focal-point position, ${ }^{5-7,13,14}$ the welding speed, ${ }^{10,12}$ or a filler wire feed rate $^{14}$ as the actuator.

Focus can be maintained with vision systems involving triangulation computation. However, the main drawback of such systems is that such off-axis measurement requires good optical access to the workpiece and necessitates some intrusion into the process. Alternative techniques based on the on-axis detection of process-generated radiation through the focusing optics and the delivery optical fiber system have been reported. One technique exploits the chromatic aberrations in the optical elements, such that 
by a spectral analysis of the detected light the focal error is derived. ${ }^{5-7}$

Postma et al. ${ }^{9,10}$ describe a power feedback control system that is capable of maintaining full penetration in mild steel sheets. The intensity of the weld pool emitted light is transmitted through the fiber back to the laser source and measured by a photodiode commercial monitoring system (WeldWatcher). The signal level is compared with that of a reference weld. However, the signal level for a partially penetrated weld can be the same as for full penetration. Therefore the process has to reach the fullpenetration regime before the controller switches on, and it can suffer from instability if a strong perturbation suddenly changes the process regime.

Another control strategy to ensure full penetration has been performed by Bagger and Olsen. ${ }^{11}$ The control is realized by a photodiode monitoring the rootside light emission from the process. It has been demonstrated that a $17-\mathrm{Hz}$ feedback control bandwidth is sufficient to maintain an even root-side signal when the speed was stepwise increased from 0.3 to $0.7 \mathrm{~m} / \mathrm{min}$ for $2-\mathrm{mm}$ sheets and when a stepwise change in the thickness sheet by lap joining 0.5 to 2 $\mathrm{mm}$ occurred. However, it requires a two-sided access, and the position of the photodiode has to be fixed relative to the laser beam, which limits the number of practical applications. In addition, the signal error level and the root reference signal have to be adjusted by factors that depend on the configuration.

In this paper we describe the design of a closed-loop system that ensures full penetration by controlling the focal position and the laser power. The focal position monitoring system is based on the chromatic aberration of the focusing optics ${ }^{5-7}$ and incorporates an automatic setup routine and a digital gain control. It is easy to use with minimal setup time and material wastage and is able to directly control the vertical stage controller. ${ }^{7}$ With the laser power control system we can analyze the degree of penetration by imaging the keyhole fluctuations with a camera. We show how the optical focus control system has been used successfully to maintain a focal position in closed loop by welding tilted and nonflat workpieces. We demonstrate also that the full-penetration recognition technique that has been developed is able to control the laser power in such a way that full penetration is maintained in the presence of a linear and step change of thickness. Finally we discuss the performances and the limitations of the systems used on a realistic aeroengine workpiece.

\section{Experimental Setup}

A Nd:YAG laser system (either TRUMPF, $4 \mathrm{~kW}$, or Rofin-Sinar, $2.5 \mathrm{~kW}$ ) was used to weld Inconel 718 and titanium (Ti-6Al-4V) plates of variable thickness (1-3 mm). All experiments were realized at a constant speed and are bead-on-plate welds. A critical aspect of welding titanium or nickel alloy is the gas protection against oxidation. Welding was therefore carried out with the workpiece inside an argon-filled closed box, ensuring good protection on both sides of

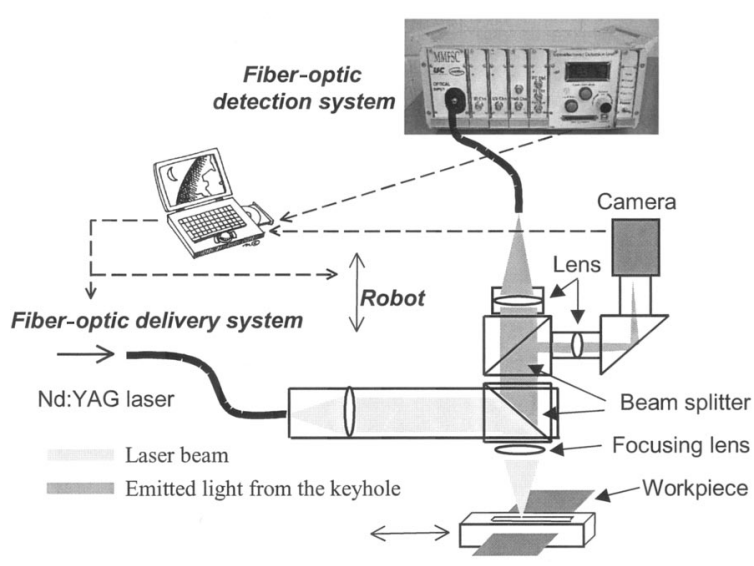

Fig. 1. Schematic of the experimental arrangement.

the workpiece. The sensor arrangement is shown schematically in Fig. 1. It is based on the coaxial detection of the light generated within the welding interaction zone, where the laser beam is focused onto the workpiece creating the welding keyhole. This keyhole light is detected by use of both a camera and a large-core optical fiber $(600-\mu \mathrm{m}$ core diameter) plus photodiodes. The fiber-optic detection system allows detection at a high temporal bandwidth but averaged over the area of the keyhole entrance, whereas the camera is used to provide a high spatial resolution but at a lower speed. Both systems are mounted directly at the welding head, and two identical beam splitters are used to collect the emitted light.

\section{Closed-Loop System Design}

\section{A. Focus Control}

The focus control technique exploits chromatic aberrations in the optical elements. The weld pool emitted light is delivered to a detection unit ${ }^{7}$ where the process radiation is split into three spectral ranges: $0.4-$ $0.7 \mu \mathrm{m}$ (UV-visible), 1.2-1.6 $\mu \mathrm{m}$ (IR), and $1.1 \mu \mathrm{m}$ (Nd:YAG). Each is focused on a different photodiode and amplified by a separate module. A further module subtracts the UV-visible signal from the IR and gives a signal related to the deviation from the optimum focus position. A real-time control signal is thus derived to drive the translation stage of the laser head focus.

The resolution is limited by noise. The principal noise source in the present experiments was the instability in the optical signals intrinsic to the oscillations of the plasma or keyhole. Previous research demonstrated that the optimum compromise between noise and bandwidth could be realized by the filtering of signals with a low-pass filter of 100 $\mathrm{Hz} .{ }^{5}$ Therefore the focal error signal was filtered at $100 \mathrm{~Hz}$.

The optical detection unit incorporates userfriendly features. The software inside provides an iterative algorithm, which takes only a few hundred milliseconds to complete, seeking the optimum amplifier gains for a specific welding condition or laser 


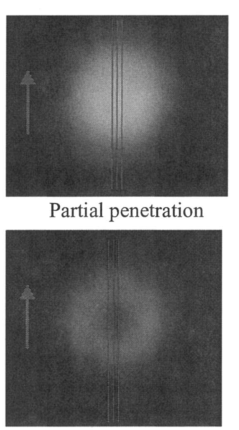

Full penetration

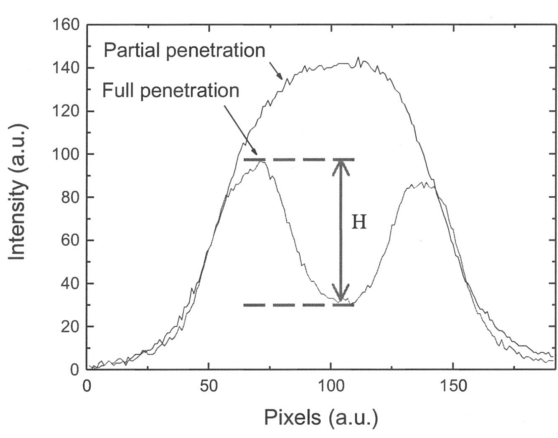

Fig. 2. Images of the keyhole and their corresponding intensity profile. The intensity profile represents the average profile along the three lines.

arrangement. In this way costly reference welds after the installation of the system can be significantly reduced. It includes analog-to-digital converters for the signals of the three channels and the focal error output, with a bandwidth of $10 \mathrm{kHz}$. A Universal Serial Bus connection to an external computer also makes it possible to record all the data and enables further signal processing in real time.

\section{B. Power Control}

The ability to detect and use the optical emissions from the weld pool area for penetration monitoring and control purposes has been examined by many authors. ${ }^{2-4}$ There exists a linear correlation between the penetration depth and the light emitted from the weld-pool area coaxially with the laser beam in the partial-penetration welding condition. In our application, the welds are required to be fully penetrating (i.e., extend completely through the thickness of the material). As specified above, a nonintrusive and on-axis method has to be used for practical reasons. We therefore investigated several full-penetration recognition techniques, based on the analysis of signals detected with both a camera and a single-point sensor to determine their relative merits. ${ }^{15}$ Some spectral and statistical analyses of the single-point sensor signals clearly indicate the presence of a fully opened keyhole, thus ensuring a fully penetrated weld. However, in our specific applications, a more robust method has been the computation of the keyhole image intensity profile along the welding path.

A complementary metal-oxide semiconductor firewire camera (Basler A602f) was used to record the keyhole images. Figure 2 shows an example of images recorded when welded in a partially penetrated and a fully penetrated regime. When the keyhole is fully penetrating, the camera image shows a circularshaped intensity minimum roughly in the center of the keyhole. The basic principle of the fullpenetration feedback control is to maintain an almost constant depth of the intensity minimum. The normalized depth coefficient $H$ is defined as follows:

$$
H=100\left(I_{\max }-I_{\min }\right) /\left(I_{\max }\right),
$$

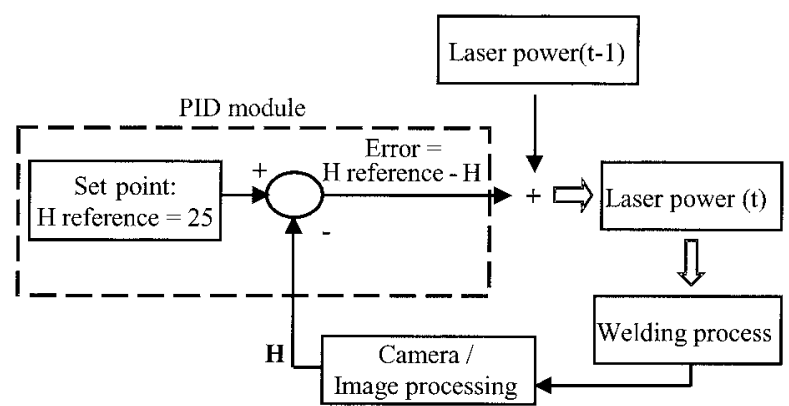

Fig. 3. Schematic of the laser power controller architecture.

where $I_{\max }$ and $I_{\min }$ are the maximum and minimum values of the intensity, respectively. The normalization avoids sensitivity to intensity changes coming from use of a different material or a change in the quality of the images (for example, because of vapor plume interference or camera blooming effect). We used a proportional-integral-derivative (PID) controller to maintain the intensity minimum depth about a specified set point by adjusting the laser power.

The architecture is shown in Fig. 3. Since the laser power bandwidth is very fast compared to the thermal response time of the process, only proportional gain was used: The controller corrects the laser power proportionally to the error of the intensity minimum depth relative to the set point. The best compromise was $H \approx 25$.

For the system, we use National Instrument's LabVIEW software that grabs the images, computes the $H$ parameter, and controls the laser power $(100-\mathrm{Hz}$ bandwidth). A 2-GHz CPU laptop was used with Windows XP.

\section{Results}

\section{A. Focus Control}

The typical focal error signal in an open-loop test is plotted in Fig. 4. This was obtained during the production of a bead-on-plate weld of Inconel when we moved the laser head position from 0 to $\pm 5 \mathrm{~mm}$. We observed similar results when welding bead-on-plate titanium workpieces. The focal error signal shows the change of the laser head position with a resolution of $\pm 0.5 \mathrm{~mm}$.

We conducted a series of experiments by tilting the workpiece at an angle of $5^{\circ}$, corresponding to a height variation of $10 \mathrm{~mm}$ over its length [Fig. 5(c)]. For open loop we observe an increase in the focal error along the weld. As long as the weld is fully penetrated, the focal error variation is linear. In the second half of the weld, full penetration is lost since conduction welding occurs, resulting in a nonrepresentative fluctuating signal. The $-2-\mathrm{V}$ shift at the start of the weld is related to a fixed offset of the laser head. In closedloop control, the focal error is maintained around zero, resulting in an excellent weld. Full penetration is ensured along the entire weld as observed [Fig. 5(b)]. Similar results were obtained with titanium. 


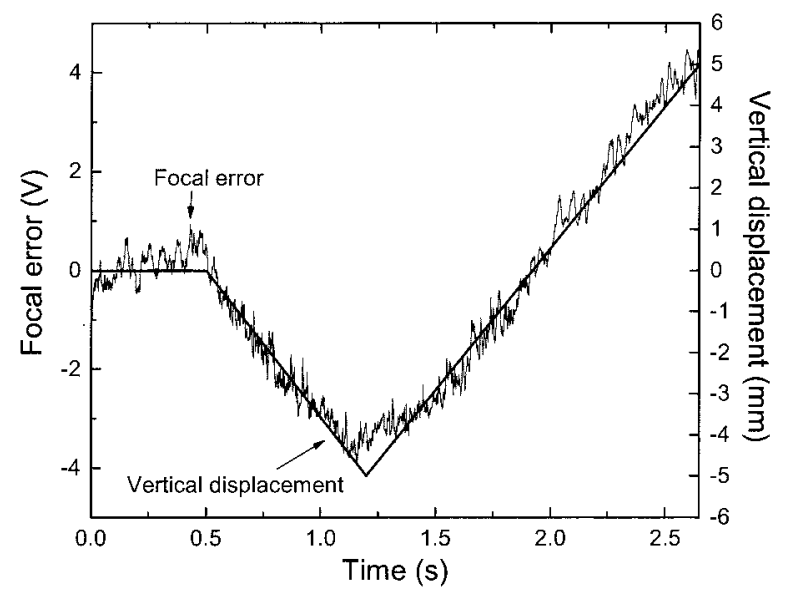

Fig. 4. Focal error signal obtained in the open-loop test when 2 -mm-thick Inconel was welded at $1.2 \mathrm{~m} / \mathrm{min}$ with $2.5 \mathrm{~kW}$ of a Nd:YAG laser beam (left-hand scale). The laser head was moved from 0 to $-5 \mathrm{~mm}$ and then to $+5 \mathrm{~mm}$ as shown on the right-hand scale.

Tests were also carried out with step changes in thickness, giving step changes in the top surface position relative to the laser. The response time for a stepwise change of $2 \mathrm{~mm}$ was $0.5 \mathrm{~s}$.

\section{B. Power Control}

With the $2.5-\mathrm{kW}$ laser, variation of the keyhole opening with laser power, as indicated by $H$, was observed for different thicknesses. Figure 6(a) shows $H$ as a function of laser power and material thickness. These

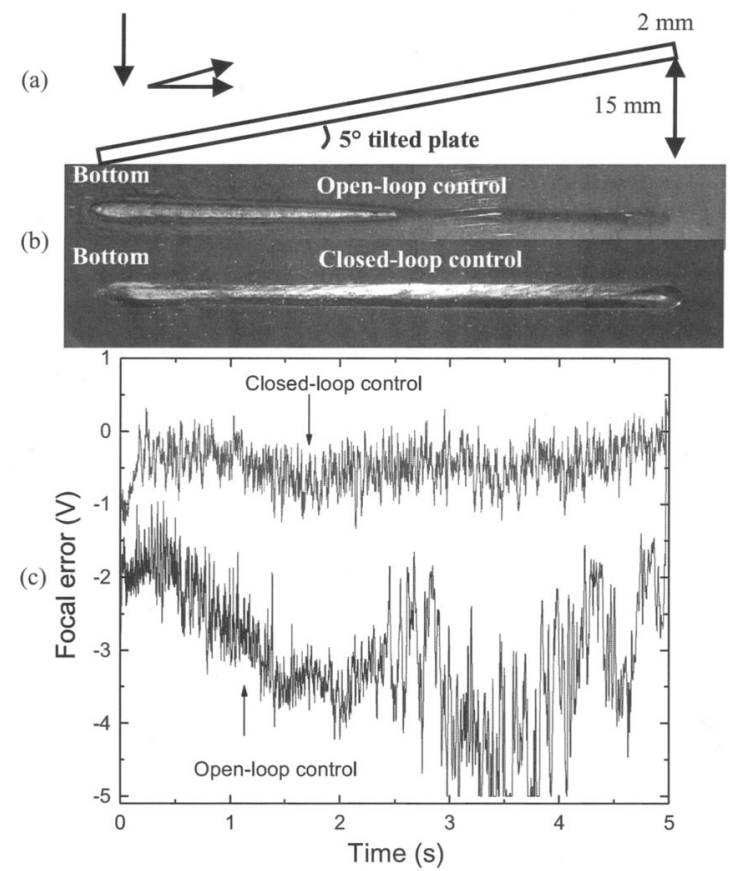

Fig. 5. Welding of a 2-mm-thick Inconel workpiece tilted at an angle of $5^{\circ}$ for a laser power of $2.5 \mathrm{~kW}$ and a speed of $1.2 \mathrm{~m} / \mathrm{min}$. (a) Longitudinal cross section, (b) photographs of rear seams obtained for welds generated with open-loop and closed-loop controls, (c) focal error signals obtained in each case.

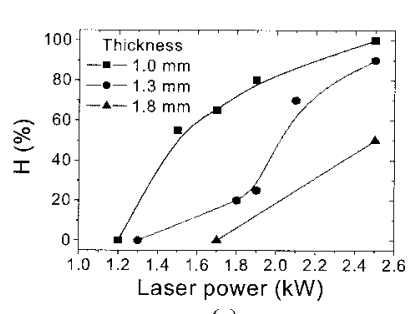

(a)

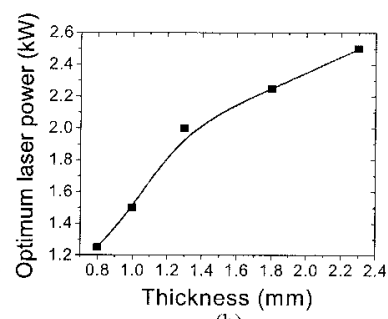

(b)
Fig. 6. (a) Keyhole opening as function of the laser power for different workpiece thicknesses. (b) Optimum laser power (i.e., giving the optimum full penetration) as a function of the workpiece thickness at a constant speed of $1.2 \mathrm{~m} / \mathrm{min}$.

data were used to tune the controller and adjust the proportional gain off line. Figure 6(b) shows the optimum laser power versus the thickness of the workpiece, revealing that the response is not linear at small thicknesses. A proportional gain of 1 was found to give a fast, smooth, and non-over-reactive response. This characterization carried out with a $2.5-\mathrm{kW}$ laser shows the general trend of the parameter $H$ as a function of laser power for a certain thickness. Although the values change with a $4-\mathrm{kW}$ laser source, this behavior is preserved. All the experiments presented below were realized with both the 2.5- and 4-kW lasers, but only the results obtained with the $4-\mathrm{kW}$ system are presented since they are similar in each case.

Two series of tests were conducted to investigate the performance of the controller in response to a linear change of thickness and then to test its limits to a step change of thickness. All the experiments were carried out from the level top surface of workpieces of variable thicknesses at a constant speed of $1.2 \mathrm{~m} / \mathrm{min}$. The laser focus was maintained throughout.

The cross section of a workpiece along the line of the weld is shown schematically in Fig. 7(a). The photograph of the top and bottom surfaces is shown in Fig. 7(b), revealing a high degree of oxidation on the top surface only. We note that our gas shielding configuration was unable to deal with very long welds (more than $110 \mathrm{~mm}$ in length), and so subsequent welds were carried out over half of the distance. The laser power signal controlled by the feedback control system and the $H$ parameter are plotted in Figs. 7(c) and $7(\mathrm{~d})$. The laser power was set to start at $3 \mathrm{~kW}$ (region 1). It results in a lack of power at this thickness $(3.1 \mathrm{~mm})$ as no opened keyhole is observed. The laser power is therefore increased from 3 to $4 \mathrm{~kW}$, revealing that at this speed the maximum laser power is not able to create a fully opened keyhole. However, it is still sufficient to obtain a fully penetrated weld. In region 2 , when the thickness abruptly decreases to $1 \mathrm{~mm}$, the laser power is rapidly adjusted to $1.8 \mathrm{~kW}$ since the depth $H$ has a peak of more than $80 \%$. It then increases roughly linearly in region 3 as the material becomes thicker to reach its maximum value (region 4).

We clearly observe that the keyhole opening is 
(a)

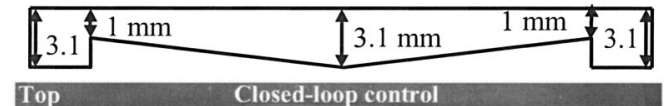

(b)

(c)

(d)

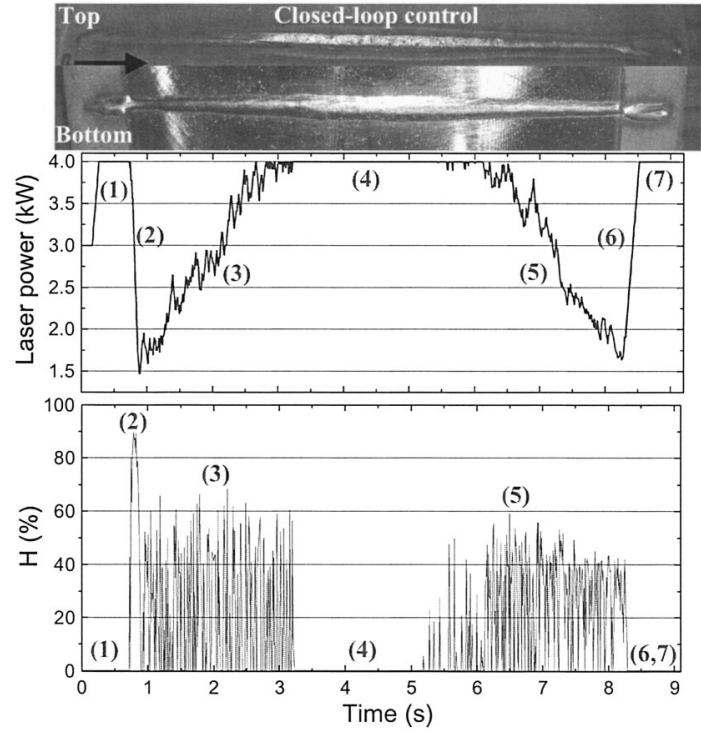

Fig. 7. Welding of a tapered and stepped Inconel workpiece for a speed of $1.2 \mathrm{~m} / \mathrm{min}$. (a) Longitudinal cross section (note that scales are different in the two axes), (b) photographs of top and bottom surfaces, (c) closed-loop laser power control signal, (d) keyhole opening parameter $H$ calculated from the image processing at $100 \mathrm{~Hz}$.

maintained close to $H=25$. The photograph of the rear side shows a visually pleasing fully penetrated weld along the entire increase of thickness. In region $4(3.1 \mathrm{~mm})$, the maximum laser power is maintained since no keyhole opening occurs. The sensor system is able to correct the laser power from 4 to $1.8 \mathrm{~kW}$ when the thickness is linearly decreased in region 5 . We note that the same value of the laser power is obtained again for a 1-mm thickness.

Several tests were carried out with the loop closed on a half-length of the workpiece to minimize oxidation, confirming the reproducibility of the control process (from thin to thick [Fig. 8(a)] and thick to thin). Use of a proportional gain of 0.5 led to a smoother but identically controlled laser power signal. This value was used for all the other experiments. Without laser power control, we note the presence of a pinhole when the weld was carried out at $4 \mathrm{~kW}$ [Fig. 8(c)]. Burnthrough was not obtained when welding occurred at a constant laser power of $3 \mathrm{~kW}$. However, the rear seam shows a thinner width for $3.1 \mathrm{~mm}$, which is not acceptable as full penetration. In addition, we also

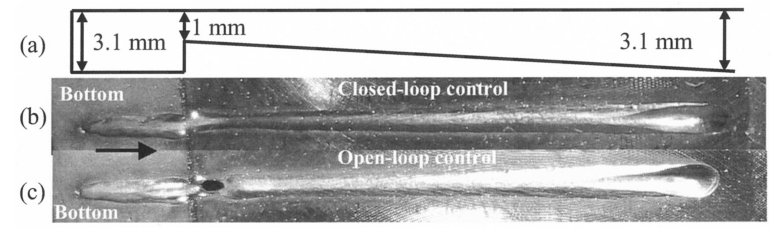

Fig. 8. Welding of a tapered and stepped Inconel workpiece for a speed of $1.2 \mathrm{~m} / \mathrm{min}$. (a) Longitudinal cross section. Photographs of bottom surfaces of a weld realized (b) with and (c) without (4-kW) closed-loop laser power control. (a)

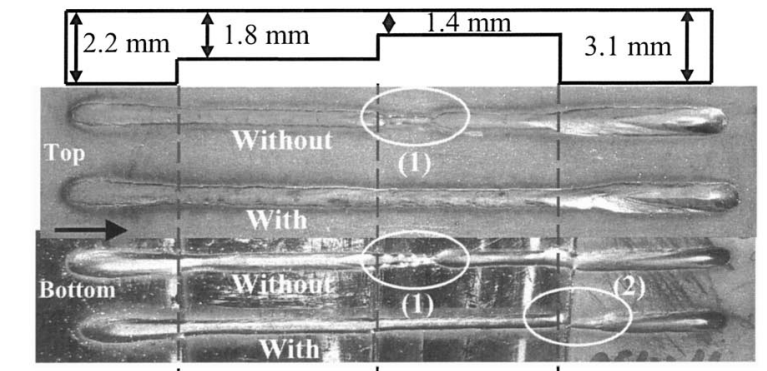

(c)

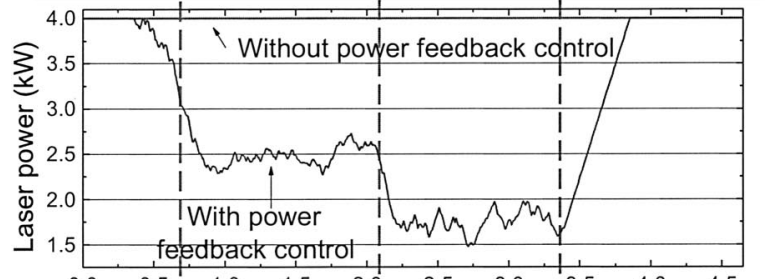

(d)

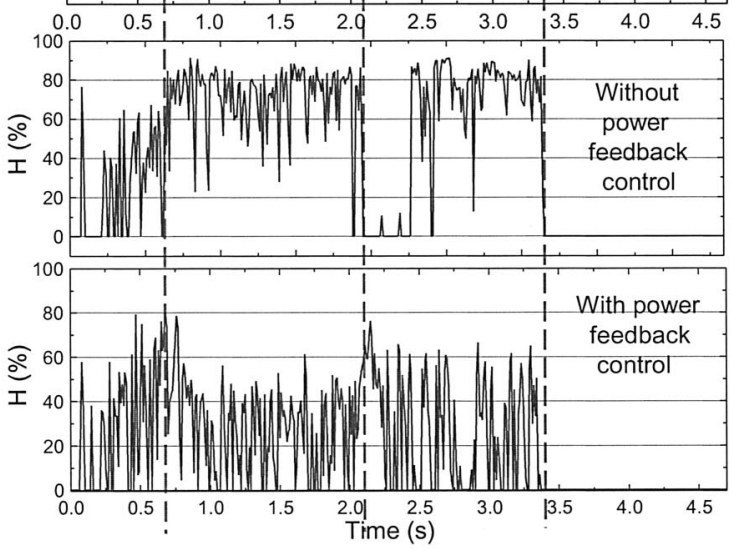

Fig. 9. Welding of a stepped Inconel workpiece at a speed of 1.2 $\mathrm{m} / \mathrm{min}$. (a) Longitudinal cross section. (b) Photographs of top and bottom surfaces of a weld realized without and with closed-loop laser power control. (c) Laser power signal controlled by the closedloop controller and without the controller (constant value of $4 \mathrm{~kW}$ ). Keyhole opening parameter $H$ for the weld realized (d) without closed-loop control and (e) with closed-loop control.

found considerable overpenetration occurring for 1 $\mathrm{mm}$. The energy surplus is dissipated in the slow increase of thickness occurring just after the abrupt change to $1 \mathrm{~mm}$. A slight change of design of the workpiece would lead to a pinhole formation as demonstrated next.

Stepped Inconel workpieces were prepared with a variety of step sizes, with the largest step change of 0.8 to $3.1 \mathrm{~mm}$. Visually stable and fully penetrating welds were obtained in the range of $2-1.1 \mathrm{~mm}$. However, the feedback control system is unable to adjust the laser power for a thickness less than $1.1 \mathrm{~mm}$, mainly because of the intrinsic instability of the weld at this speed and thickness.

Figure 9 shows the effect of successive step changes of thickness: $2.2,1.8,1.4$, and $3.1 \mathrm{~mm}$. Two welds were carried out; one was realized at a constant laser power of $4 \mathrm{~kW}$ that is the optimum laser power for the 2.2-mm-thick part of the workpiece, whereas we carried out the second by adjusting the laser power with the feedback control system.

Photographs of the top and bottom surfaces of the 
weld carried out at a constant power of $4 \mathrm{~kW}$ are shown in Fig. 9(b). For the 1.4-mm-thick section, in this case a hole was not produced as might have been expected, but instead a weld with a much reduced width is created. Its corresponding opening keyhole depth is plotted in Fig. 9(d). It shows that an overpenetration occurs for the thickness of $1.8 \mathrm{~mm}(H$ $\approx 80 \%$ ), for the 1.4-mm-thick part, $H$ abruptly falls to zero and rises again to $80 \%$. The absence of a hole means that, although the majority of the laser power passed through the workpiece, the expanded keyhole was not too large and the surrounding molten material rejoined. We comment on this behavior in Section 5. Generally, we found that a burn-through weld occurs at a thickness of $\leq 1.4 \mathrm{~mm}$ with a constant laser power of $2.8 \mathrm{~kW}$ (optimum laser power for the thickness of $2 \mathrm{~mm}$ ). Figure 9(c) shows that the feedback controller has successfully adjusted the laser power from $4 \mathrm{~kW}(2.2 \mathrm{~mm})$ to $2.4 \mathrm{~kW}(1.8 \mathrm{~mm})$, then to 1.4 $\mathrm{kW}(1.4 \mathrm{~mm})$, and eventually back to $4 \mathrm{~kW}$ again (3.1 $\mathrm{mm}$ ). We kept the keyhole opening parameter around 25 as shown in Fig. 9(e) by adjusting the laser power. There is, however, a slight loss of full penetration at the interface between 1.4 and $3.1 \mathrm{~mm}$ (region 2). This is not observed for the constant laser power weld [Fig. 9 (b)] because of the overpenetration occurring for this thickness.

\section{Focus and Power Control}

Figure 10 shows the results obtained with a $5^{\circ}$ tilted workpiece and a stepped thickness change of $2.1,1.5$, 1.1 , and $3.1 \mathrm{~mm}$, both with and without focus and power feedback control. Photographs [Fig. 10(b)] at a constant laser power of $2.8 \mathrm{~kW}$ (giving the optimum full penetration for $2.1 \mathrm{~mm}$ ) show that, despite the defocused beam, the energy is still sufficient to form a hole in the 1.1-mm-thick part. In addition, the beam becomes so unfocused [Fig. 10(d)] at a 3.1-mm thickness that full penetration is lost. We can clearly see that both feedback controls are required in this configuration. If control is used, results shown in Fig. 10(c) are obtained demonstrating that the full penetration is maintained whatever the change of thickness and focus. No hole is observed and a visually nice weld is obtained along the entire length. Nevertheless, we can see a slight loss of full penetration at the step from 1.1 to $3.1 \mathrm{~mm}$ as above. As plotted in Fig. 10(d), the focal error stays around zero. The vertical displacement of the laser head recorded by the translation stage confirms that it has followed the surface of the workpiece since a vertical upwards movement of $10 \mathrm{~mm}$ has occurred that corresponds to the displacement created by the tilt. We observe that for each thickness, the laser power signal controlled by the system is stable and smooth.

Another series of experiments was carried out on a realistic airfoil shape, made of Inconel 718 [Fig. 11(a)]. The weld started and ended on a 3-mm-thick Inconel workpiece tack welded on each of the airfoil edges. We obtained a visually good weld, although it was slightly oxidized on its top surface [Fig. 11(c)]. The laser head was maintained at optimum focus. The vertical dis- (a)

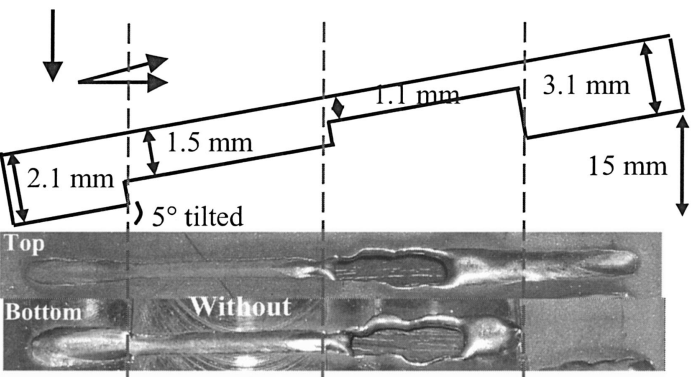

(b)

(c)

(d)
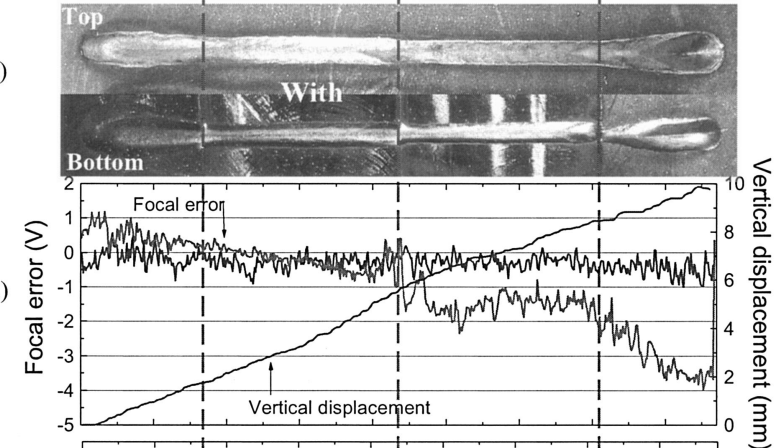

(e)

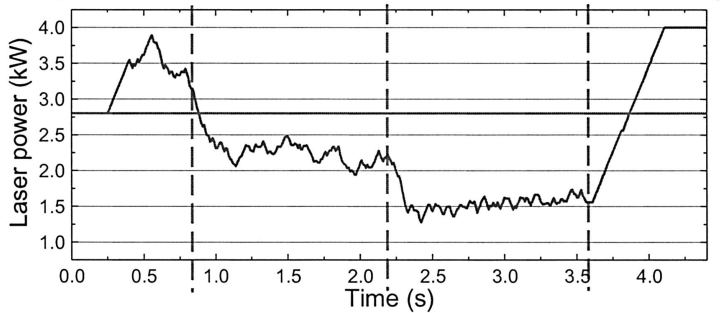

Fig. 10. Welding of a stepped Inconel workpiece tilted at an angle of $5^{\circ}$ for a speed of $1.2 \mathrm{~m} / \mathrm{min}$. (a) Longitudinal cross section. (b) Photographs of top and bottom surfaces of the weld without closedloop focus and laser power control (constant value of $2.8 \mathrm{~kW}$ ). (c) Photographs of top and bottom surfaces of the weld in the closedloop control regime (focus and laser power). (d) Focal error of uncontrolled and controlled weld (left-hand scale) and displacement of the laser head recorded by the vertical translation stage during the focus control weld (right-hand scale). (e) Laser power signal for both welds.

placement measured by the stage controller shows that the laser head has followed a similar trajectory to the measured airfoil height [Fig. 11(e)], also confirmed by the focal error signal. The laser power maintained at $4 \mathrm{~kW}$ when welding the 3 -mm-thick part decreases to $2.4 \mathrm{~kW}$ when welding on the airfoil. The power stabilizes at around $2.5 \mathrm{~kW}$ and eventually increases at the end of the airfoil to reach $4 \mathrm{~kW}$ again when reaching the 3-mm-thick part. The bottom side of the weld confirms that the weld is fully penetrated even on the edge where the thickness increases from 2 to at least 5 $\mathrm{mm}$ when the top and bottom parts merge [as shown in Fig. 11(b)].

\section{Discussion and Conclusion}

The range over which focus can be maintained is limited only by the traverse range of the motorized stage, provided that the focus distance changes at a speed within the capabilities of the stage and the bandwidth of the feedback loop. The practical band- 


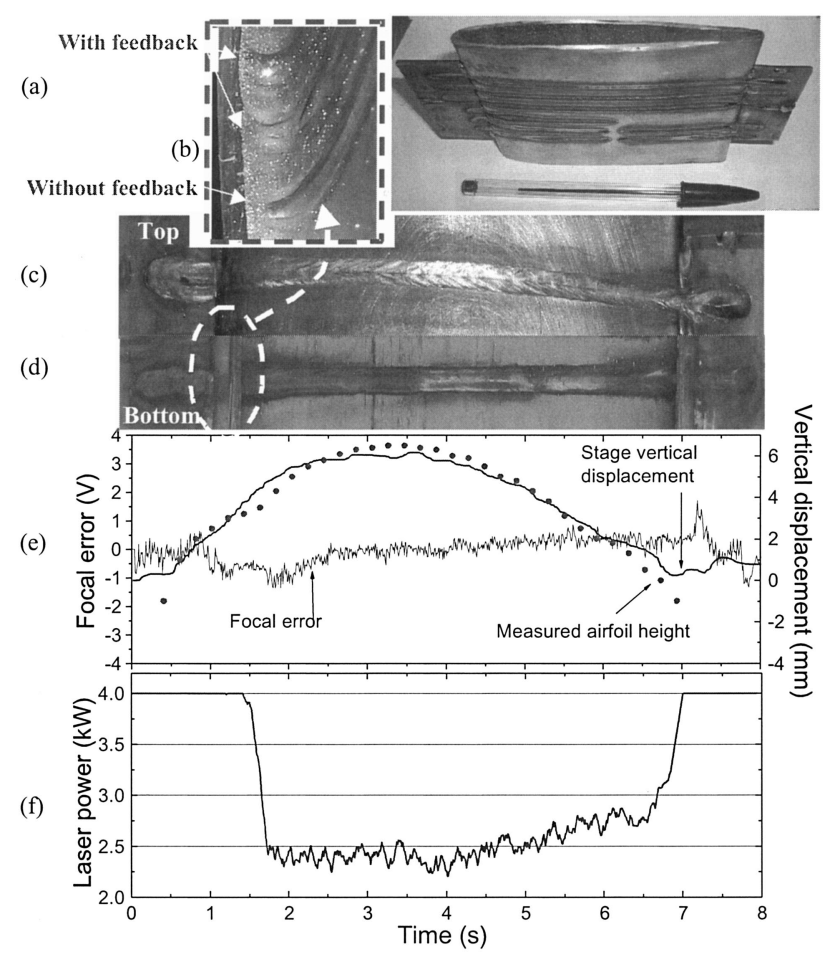

Fig. 11. Welding of an Inconel airfoil for a speed of $1.2 \mathrm{~m} / \mathrm{min}$. (a) Photograph of the entire airfoil. (b) Photograph of left internal airfoil edge. Photographs of (c) top and (d) bottom surfaces of the weld. (e) Focal error signal obtained in the closed-loop system (left-hand scale) and vertical displacement of the laser head recorded by the vertical translation stage and measure of the height change of the top surface of the airfoil (right-hand scale). (f) Closedloop laser power control signal.

width of the system, limited by the response of the vertical motorized stage, was approximately $30 \mathrm{~Hz}$. We set the bandwidth of the optical detection unit to $100 \mathrm{~Hz}$ using a low-pass filter. It was possible to maintain the optimum focal position, ensuring full penetration at typical speeds of $1.2 \mathrm{~m} / \mathrm{min}$ for Inconel and $1.8 \mathrm{~m} / \mathrm{min}$ for titanium. The tested configurations show that the focus control system is able to adjust the focus by $10 \mathrm{~mm}$ over a distance of $100 \mathrm{~mm}$ for Inconel and titanium.

Penetration feedback control is based on image processing to analyze the degree of opening of an opened keyhole. ${ }^{15}$ An interesting phenomenon was observed during the formation of a hole, occurring when we used high laser power on a thin workpiece. The keyhole images show a keyhole profile depth becoming very large, reaching $100 \%$ for few milliseconds. A completely black image corresponding to a total penetration of the light through the plate can be obtained. A few milliseconds later, if the laser power is kept constant, the keyhole images change aspect and become similar to what we obtained when welding in a partial-penetration regime, i.e., a single peak intensity profile. However, a large hole is obtained along the entire weld [Fig. 10(b)]. We suggest that the light may come from the molten pool emission that hides the total collapse of the keyhole. We can easily understand the drawback of such behavior for a control system. It is therefore necessary for the system to react quickly enough to avoid the formation of the hole. For the case in which the majority of the laser power passed through the workpiece without creation of a hole (Fig. 9, region 1), an almost black image is observed ( $H$ is null), which we wrongly interpreted as no laser light reaching the workpiece. A later version of the algorithm takes this phenomenon into account since the laser controller input is used to indicate whether light is being emitted from the laser. The $H$ coefficient in Fig. 9(d) (region 1) is thus corrected and oscillates between $100 \%$ and $0 \%$ (instead of $0 \%$ ). Oscillations are due to the alternation between a complete black image and a partial black image (with a single peak wrongly interpreted as a partial penetration). As with hole formation, it is thus necessary for the system to avoid overpenetration before it is fully formed.

The bandwidth is limited by the sampling rate of the camera and the processing speed since the response time of the Nd:YAG laser is very fast. Practically, the feedback control has been realized at 100 $\mathrm{Hz}$, which is the limit for both the sampling rate and the processing speed by use of standard complementary metal-oxide semiconductor firewire camera and a 2-GHz CPU laptop under Windows XP software. This non-real-time hardware has been proved to be fast enough to maintain a real-time record of images while data are acquired or generated from two analog inputs at $110 \mathrm{~Hz}$ and one output at $100 \mathrm{~Hz}$ for a process time of at least $30 \mathrm{~s}$.

Experimental results achieved from a lessexpensive camera with a maximum frame rate of 30 $\mathrm{Hz}$ (Unibrain Fire-i400) also demonstrated that a bandwidth of $30 \mathrm{~Hz}$ was still effective to maintain the optimum full penetration when we welded bead on plate with a progressive change of thickness from 3 to $1 \mathrm{~mm}$. However, the signal response was smoother when we were controlling at $100 \mathrm{~Hz}$, especially for step change thickness.

The thermal response of the process at the start of the weld leads to an overreaction of the feedback laser power control. Therefore a small delay of $0.25 \mathrm{~s}$ was implemented in the control algorithm to allow a stabilized keyhole to form when the feedback control is switched on. Its effect is visible on the laser power value in Figs. 7(c), 9(c), and 10(e) since the power is maintained constant for $0.25 \mathrm{~s}$ at each weld beginning. It corresponds to a thermal response of $4 \mathrm{~Hz}$.

A PID control system is designed to be effective in a linear regime. As shown in Fig. 6(b), the optimum laser power response is not a linear function of the workpiece thickness, especially in the thinner range. However, the PID-based feedback control system that we developed is stable over a very wide range of thicknesses from 3 to $1.1 \mathrm{~mm}$. Below $1.1 \mathrm{~mm}$ it becomes unstable and is unable to avoid burn-through. However, we note that welds with a workpiece thickness of less than $1 \mathrm{~mm}$ at $1.2 \mathrm{~m} / \mathrm{min}$ were unstable whatever the laser power. Such thin workpieces must be welded at a faster speed, a parameter that was not 
controlled by our system (but which could potentially be controlled). It confirms that the nonlinear behavior of the process stays in the acceptable limit of the designed control system.

The main issue that has been observed is the slight loss of penetration occurring for a change of thickness from 1.1 to $3.1 \mathrm{~mm}$ (i.e., $2-\mathrm{mm}$ change). It is due to the response time of the feedback control. It can be minimized by an increase in the proportional gain to obtain a faster response, but this can give rise to oscillations. However, for a more realistic value of a 1 -mm step, penetration is maintained without an increase in the gain. A key measure of the robustness of a control system is its stability over a wide range of defects for constant PID coefficients. In addition, the system has demonstrated its ability to see a change of thickness. If the different thicknesses of the workpiece are known, the algorithm can be easily programmed to switch to a preset value of the laser power. On the other hand, changing the design with a bevel can overcome this drawback. Another limitation of the system is that it is unable to distinguish between a full-penetration weld obtained without an opened keyhole and a partially penetrated weld (as shown in Fig. 7 in regions 1,4 , and 7) since $H$ is null in both cases. This limits the range of thicknesses (or speeds) that the system can deal with to maintain full penetration.

We find again the same ability of control when both control systems are used at the same time. Indeed the power control is dependent on the quality of the image, and hence of the focus control. A critical issue is to maintain control of the focus position because a focal error of more than $2 \mathrm{~mm}$ can lead in a wrong recognition process of the keyhole images. The focus control system has also proved to be effective on the bead-on-plate welding of an airfoil, inducing a displacement of almost $6 \mathrm{~mm}$ up and $6 \mathrm{~mm}$ down in $2 \mathrm{~s}$ at a speed of $1.2 \mathrm{~m} / \mathrm{min}$ (Fig. 11). In particular, it drastically reduces the setup time to program the trajectory close to the shape of the part. As shown in Fig. 11, optimum full penetration has been maintained along the entire weld. However, although the penetration control on the middle part has proved to be good for all our tests, a small burn-through has been observed for the majority of the tests at the edge of the airfoil where the two sheets are less than $2 \mathrm{~mm}$ apart, i.e., the right edge of the airfoil in Fig. 11(a). At the other edge [left edge of the airfoil in Fig. 11(a)] the free space is somewhat larger $(>2 \mathrm{~mm})$, and no pinhole is formed. Observation of the keyhole images does not, however, show any evidence of this hole. The hole arises because of the close presence of the back face of the airfoil; the light penetrating through the front face of the airfoil is reflected by the close back face of the airfoil, adding light in the central region of the keyhole image. It results in a hidden overpenetration state and an incorrect interpretation by the penetration control system that increases the laser power too much. It clearly shows a limitation of the system, as it is relevant to the geometry of the workpiece. However, it may be possible to combine our technique with spectral analysis of the signals from the optical detection unit to correct this failure; a clear frequency peak in the $1-3-\mathrm{kHz}$ range is observed when one is welding in the full-penetration regime. ${ }^{15}$ In general, as the control system is based on image processing, recognition performance can suffer from a degradation of the image quality coming from an error of focus, plume interference, and oxidation.

To conclude, a real-time, nonintrusive focus and laser power control system for laser welding of aeronautic components with a Nd:YAG laser has been successfully demonstrated to ensure an optimum full penetration. Focus control was achieved over a wide range of welding parameters and has been proved to be able to maintain the focus position in a real welding situation without any interference from the laser power change. We achieved power control by a simple, low-cost keyhole image recognition system by analyzing the degree of the opening of the keyhole in a fully penetrated keyhole welding at $100 \mathrm{~Hz}$. It can maintain full penetration over a wide range of changes in workpiece thicknesses at a constant welding speed of $1.2 \mathrm{~m} / \mathrm{min}$, avoiding the burn-through or lack of penetration that is obtained if a constant laser power is applied. Potential applications can be found in real workpieces such as airfoils that can have a nonconstant thickness either due to geometry (e.g., double thickness close to the edges) or due to casting repair work. It should allow a drastic reduction in the time required to optimize the laser power in such configurations. It would be useful to carry out further investigations into the response of the system to a change of speed or its operation in a butt-joint welding regime.

The authors acknowledge the support of the European Commission, which has provided enabling funds through the project Manufacturing and Modeling of Fabricated Structural Components. We are also grateful to A. Campo and J. L. Rodríguez for their technical input and to Manuel Trucco for fruitful discussions.

\section{References}

1. J. Beersiek, "New aspects of monitoring with a CMOS camera for laser materials processing," in Proceedings of the International Congress on Applications of Lasers and Electro-Optics (Laser Institute of America, Orlando, Fla., 2002).

2. P. Abels, S. Kaierle, C. Kratzsch, R. Poprawe, and W. Schulz, "Universal coaxial process control system for laser material processing," in Proceedings of the International Congress on Applications of Lasers and Electro-Optics (Laser Institute of America, Orlando, Fla., 1999), Sect. E, pp. 99-108.

3. J. Beersiek, R. Poprawe, W. Schulz, H. Gu, R. E. Mueller, and W. W. Duley, "On-line monitoring of penetration depth in laser beam welding," in Proceedings of the International Congress on Applications of Lasers and Electro-Optics (Laser Institute of America, Orlando, Fla., 1997), Vol. 83, Part 1, Sect. C, pp. 30-39.

4. M. Dahmen, S. Kaierle, P. Abels, C. Kratzsch, E. W. Kreutz, and R. Poprawe, "Adaptive quality control for laser beam welding," in Proceedings of the International Congress on Applications of Lasers and Electro-Optics (Laser Institute of America, Orlando, Fla., 1999), Sect. D, pp. 29-38. 
5. F. M. Haran, D. P. Hand, C. Peters, and J. D. C. Jones, "Focus control system for laser welding," Appl. Opt. 36, 5246-5251 (1997).

6. M. D. T. Fox, D. P. Hand, D. Su, J. D. C. Jones, S. A. Morgan, M. A. McLean, and W. M. Steen, "Optical sensor to monitor and control temperature and build height of the laser direct-casting process," Appl. Opt. 37, 8429-8433 (1998).

7. A. Cobo, F. Bardin, P. Aubry, W. Knapp, O. Collin, J. D. C. Jones, D. P. Hand, and J. M. López-Higuera, "Optical fibrebased focus control system for laser welding incorporating automatic setup," in Proceedings of the Sixteenth International Conference on Optical Fiber Sensors (Institute of Electronics, Information and Communications Engineers, Tokyo, 2003), pp. $412-415$.

8. C. Dietz, M. Jurca, L. Schlichtermann, M. Kigel-Hollacher, S. Breitschwerdt, C. Schmid, and L. Rowold, "Closed loop control system for laser welding of transmission parts," in Proceedings of the International Congress on Applications of Lasers and Electro-Optics (Laser Institute of America, Orlando, Fla., 1998), Vol. 85, Sect. C, pp. 178-186.

9. S. Postma, R. G. K. M. Aarts, J. Meijer, and B. Jonker, "Penetration control in laser welding of sheet metal," J. Laser Appl. 14, 210-214 (2002).

10. S. Postma, R. G. K. M. Aarts, J. Meijer, and B. Jonker, "Feedback control for optimal production speed in laser beam weld- ing of mild steel," in Proceedings of the International Congress on Applications of Lasers and Electro-Optics (Laser Institute of America, Orlando, Fla., 2002).

11. C. Bagger and F. O. Olsen, "Laser welding closed-loop power control," J. Laser Appl. 15, 19-24 (2003).

12. H. Derouet, L. Sabatier, F. Coste, and R. Fabbro, "Process control applied to laser surface remelting," in Proceedings of the International Congress on Applications of Lasers and Electro-Optics (Laser Institute of America, Orlando, Fla., 1997), Vol. 83, Part 1, Sect. C, pp. 85-92.

13. H. Gu and W. W. Duley, "A novel detector for closed-loop focus control during laser beam welding," in Proceedings of the International Congress on Applications of Lasers and ElectroOptics (Laser Institute of America, Orlando, Fla., 1998), Vol. 85, Sect. C, pp. 69-75.

14. H. K. Tonshoff, A. Ostendorf, and W. Specker, "Quality assurance of laser welding processes by adaptive closed-loop process control," in Proceedings of the International Congress on Applications of Lasers and Electro-Optics (Laser Institute of America, Orlando, Fla., 2000), Sect. E, pp. 252-261.

15. F. Bardin, A. Cobo, J. M. Lopez-Higuera, O. Collin, P. Aubry, T. Dubois, M. Högström, P. Nylen, P. Jonsson, J. D. C. Jones, and D. P. Hand, "Optical techniques for real-time penetration monitoring for laser welding," Appl. Opt. (submitted for publication). 